\title{
Assessing outcomes of enhanced chronic disease care through patient education and a value-based formulary study (ACCESS) - study protocol for a $2 \times 2$ factorial randomized trial
}

David J. T. Campbell ${ }^{1}$, Marcello Tonelli ${ }^{2}$, Brenda Hemmelgarn², Chad Mitchell ${ }^{3}$, Ross Tsuyuki ${ }^{4}$, Noah Ivers ${ }^{5}$, Tavis Campbell ${ }^{6}$, Raj Pannu', Eric Verkerke ${ }^{7}$, Scott Klarenbach ${ }^{8}$, Kathryn King-Shier ${ }^{9}$, Peter Faris ${ }^{10}$, Derek Exner ${ }^{2}$, Vikas Chaubey ${ }^{11}$, Braden Manns ${ }^{2,12^{*}}$ (i) and On behalf of the Interdisciplinary Chronic Disease Collaboration

\begin{abstract}
Background: Chronic diseases result in significant morbidity and costs. Although medications and lifestyle changes are effective for improving outcomes in chronic diseases, many patients do not receive these treatments, in part because of financial barriers, patient and provider-level knowledge gaps, and low patient motivation. The Assessing outcomes of enhanced chronic disease care through patient education and a value-based formulary study (ACCESS) will determine the impact of two interventions: (1) a value-based formulary which eliminates copayment for high-value preventive medications; and (2) a comprehensive self-management support program aimed at promoting health behavior change and medication adherence, combined with relay of information on medication use to healthcare providers, on cardiovascular events and/or mortality in low-income seniors with elevated cardiovascular risk.

Methods: The ACCESS study will use a parallel, open label, factorial randomized trial design, with blinded endpoint evaluation in 4714 participants who are over age $>65$ (and therefore have drug insurance provided by Alberta Blue Cross with $30 \%$ co-payment); are at a high risk for cardiovascular events based on a history of any one of the following: coronary heart disease, prior stroke, chronic kidney disease, heart failure, or any two of the following: current cigarette smoking, diabetes mellitus, hypertension, or hypercholesterolemia; and have a household income $<$ Can $\$ 50,000$. This 3 -year study is powered to detect a minimal clinically important relative risk reduction of $12 \%$ in the composite clinical outcome of all-cause mortality, nonfatal myocardial infarction, nonfatal stroke, need for coronary revascularization, and hospitalizations for chronic disease-related ambulatory care sensitive conditions, each of which will be assessed using healthcare administrative data. Secondary outcomes will include quality of life and healthcare costs.

(Continued on next page)
\end{abstract}

\footnotetext{
* Correspondence: Braden.Manns@albertahealthservices.ca

${ }^{2}$ Departments of Medicine and Community Health Sciences, Libin Institute and Institute for Public Health, Cumming School of Medicine, University of Calgary, Calgary, Canada

${ }^{12}$ Foothills Medical Centre, 1403 29th St. NW, Calgary, Alberta, CanadaT2N 2T9

Full list of author information is available at the end of the article
} 


\begin{abstract}
(Continued from previous page)
Discussion: Given identified gaps in care in chronic disease, and the frequency of financial and knowledge-related barriers in low-income Albertans, this study will test the impact of providing free high-value preventive medications (i.e., value-based insurance) and a tailored self-management education and facilitated relay strategy on outcomes and costs. By measuring the impact on both health outcomes and costs, as well as the impact on reducing health inequities in this vulnerable population, our study will facilitate informed policy decisions.
\end{abstract}

Trial registration: Clinicaltrials.gov: NCT02579655. Registered Oct 15, 2015.

Keywords: Randomized clinical trial, Chronic disease, Cost, Education, Drug insurance

\section{Background}

The World Health Organization estimates that nearly one third of worldwide deaths are from cardiovascular disease [1]. Approximately $80 \%$ of these are from myocardial infarctions and strokes. Similar statistics have been demonstrated in Canada [2]. Important risk factors for cardiovascular events include prior stroke or myocardial infarction, chronic kidney disease, heart failure, diabetes, or hypertension (henceforth referred to as "chronic diseases").

Health behavior changes (e.g., exercise, healthy diet, smoking cessation, and adherence with pharmacologic preventive management) are particularly important in the management of people with these chronic diseases [3-7]. We identified a significant evidence to care gap for people with chronic diseases in Alberta, Canada, similar to that observed in other North American populations, including that nearly $50 \%$ of people with cardiovascular-related chronic diseases do not take guideline-recommended medications (e.g., statins) [8].

As occurs in nearly all organizations for economic cooperation and development countries [9], most Canadian citizens (irrespective of whether they have private or publicly funded drug insurance) are subject to cost-sharing (i.e., copayments or deductibles) [10]. This requires that patients pay a portion of the medication cost-typically a copayment of $20-30 \%$ of the total cost of the prescription [11] —or deductibles which may be as high as $5-20 \%$ of household income [12]. In a recent survey of nearly 2000 Western Canadians, up to $20 \%$ of Western Canadian patients identified having financial barriers to obtaining their drugs; and patients with these barriers were $30-55 \%$ less likely to use statins than those who did not perceive financial barriers [10].

In addition to financial barriers, in our recent survey of Western Canadians, we also identified that patient and provider-level knowledge gaps and patient motivation were major barriers to optimal medical management [13]. Patient knowledge and motivation both tie into the concept of self-management [14]: a set of positive health behaviors which include adherence to prescribed medications. Our recent survey suggested that $40 \%$ of patients with these chronic conditions did not recall receiving health behavior change counseling, and of those who did, up to $70 \%$ of them did not report following the advice given [13].

Management of chronic disease may be suboptimal when patients experience financial barriers or do not engage in self-management. If suboptimal control persists for extended periods, patients may be hospitalized for complications [15] such as myocardial infarction, stroke, coronary revascularization, or chronic disease-related ambulatory care sensitive conditions (ACSC). ACSC are those for which "timely and effective outpatient care, including use of appropriate medications, can reduce the risk of hospitalization" [16]. These potentially preventable hospitalizations are clinically relevant to patients and for health systems [17-19].

\section{Rationale for study interventions}

a) Value-based formulary which eliminates copayment for high-value preventive medications

One way to reduce the frequency of financial barriers is to provide full coverage for "high-value" medications. Also known as value-based insurance, within a value-based formulary, medications shown to confer important benefits in high quality studies, and/or provide good value for money are provided for free, with other lower-value medications still subject to patient copayment [20]. Numerous observational studies have suggested that reducing copayments has the potential to improve adherence [20], and a recent high-quality randomized clinical trial (MI-FREEE) has demonstrated that eliminating copayments for betablockers, angiotensin converting enzyme inhibitors, statins, and antiplatelet agents in patients post-myocardial infarction reduced the total number of cardiovascular events [21]. The impact of value-based insurance in a broader patient population has not been tested using a rigorous study design. The ACCESS study is powered to determine the impact of value based insurance in a broader patient population on both clinical outcomes and costs-and as such will address an important gap in the literature. 
b) Self-management support program

A variety of interventions to enhance patient selfmanagement have been tested in people with chronic diseases within randomized clinical trials [22]. Within a recent systematic review that sought to determine which elements of such programs were most effective in people with diabetes, interventions to enhance self-management, patient education, and facilitated relay of information to clinicians were noted to be some of the most effective interventions at improving glycemic control and blood pressure [22]. "Facilitated relay" is defined as clinical information transmitted to clinicians by means other than the existing medical record [22]; the expectation is that clinicians act on the information to improve patient management. The systematic review noted considerable heterogeneity in the observed efficacy of the selfmanagement programs. Part of this difference may relate to the format of the message, which (when crafted and delivered by health care workers) may lack effective techniques demonstrated to promote behavior change, and may not be tailored appropriately to individuals' preferences and values [23]. Additional uncertainty with respect to the effectiveness of interventions to enhance selfmanagement, patient education, and facilitated relay of information to clinicians was also noted because of low sample sizes and short study duration (almost all studies $<6$ months) [22]. This uncertainty will be addressed by the ACCESS study, which will be powered to detect differences in clinical and cost outcomes.

\section{Objectives}

The Assessing outcomes of enhanced chronic disease care through patient education and a value-based formulary study (ACCESS) will determine the effectiveness of two interventions targeting financial and self-management related barriers:

(i) a value-based formulary that eliminates co-payment for select high-value medications (i.e., value based insurance); and

(ii)a comprehensive self-management support program aimed at promoting health behavior change (by addressing concerns and improving self-efficacy), combined with relay of information on medication use to health care providers on cardiovascular events and/ or mortality in low-income seniors with elevated cardiovascular risk.

\section{Trial design}

ACCESS is a pragmatic, parallel group, open-label, factorial randomized controlled trial with blinded endpoint evaluation of the two interventions described above. In this $2 \times 2$ design, there will be four treatment arms with an allocation ratio of 1:1:1:1. We will assess the impact of these interventions on both relevant clinical outcomes and healthcare system costs over a 3-year follow-up period.

\section{Methods \\ Study setting}

The ACCESS trial will recruit community-based participants living in Alberta, the fourth largest Canadian province with a population of 4.2 million [24]. In Canada, universal public health insurance provides physician and hospital services free of charge to all citizens and residents, but Alberta government-sponsored drug insurance is only provided without premium to people receiving social assistance and those over the age of 65 years. Those over age 65 pay $30 \%$ copayment for drugs (to a maximum of $\$ 25$ per prescription).

\section{Eligibility criteria}

Inclusion criteria include: (a) age greater than 65 years with Alberta government-sponsored seniors drug insurance (30 \% copayment), (b) high cardiovascular risk based on a history of any one of: heart disease, stroke, chronic kidney disease, heart failure, or any two of: current smoking, diabetes, hypertension, or high cholesterol; and (c) household income $<\$ 50,000$.

Exclusion criteria include: (a) coverage by a secondary insurance plan (in addition to Blue Cross), resulting in patient-borne copayment of $<30 \%$, or (b) inability to participate in self-management modules due to cognitive impairment or a lack of an English-speaking family member or close friend.

\section{Goals of interventions}

The overarching purposes of the interventions in the ACCESS study will be to:

(1)Increase initiation and adherence to medications that have been proven to reduce the risk of cardiovascular events in this population of high risk patients, including HMG-CoA reductase inhibitors (Statins) [25] and renin-angiotensin-aldosterone system inhibitors (ACE inhibitors [ACEi] and angiotensin receptor blockers [ARBs]) [26].

(2)Encourage participants to make positive health behavior changes, including healthy dietary choices, engagement in physical activity, cessation of tobacco use, and increased adherence to all preventive medications.

Intervention 1 will be enrolment in a new drug formulary (operationalized through their existing government drug insurance) that will eliminate copayments for high value preventive medications (those which prevent myocardial infarction, strokes, hospitalizations and delay 
progression of kidney and other vascular disease): statins, beta blockers, ACE-i, ARBs, calcium channel blockers, diuretics, anti-platelet agents, anti-arrhythmic drugs, anticoagulants, oral anti-diabetes agents, insulins, and smoking cessation aids (Additional file 1: Appendix 1).

Intervention 2 will be a tailored, adaptive, and interactive self-management support system that attempts to address determinants of intention and self-efficacy with regards to improving health habits and medication taking behavior. The second component of this intervention is comprised of facilitated relay of participants' current use of recommended medications to their regular healthcare providers to facilitate prescription changes as needed, with the goal of overcoming clinical inertia.

The system was designed by behavioral and implementation scientists, clinical experts, health services researchers, in partnership with a health marketing firm (Emergence, New York, NY) and a health technology firm (Locus Health, Charlottesville, VA) to incorporate principles of design-thinking and brand-engagement, informed by a process of patient engagement, as described below. Details, including the final design and implementation plan, are presented in the Box.

\section{Box: Intervention 2 design and implementation}

Step 1-defining the objective: the primary objective of intervention 2 was to improve patient knowledge and participants' selfmanagement abilities including making positive health behavior changes including adherence to prescribed medications (especially statins and ACE-inhibitors/ARBs), healthy lifestyle choices, and smoking cessation for those who self-report smoking cigarettes. Step 2-initial patient engagement: we conducted a series of ethnographic-style interviews where one academic researcher (DC, BM, TC) was paired with a marketing expert to visit the homes of ten purposively sampled community dwelling patients chosen on the basis of having low incomes and several chronic conditions that would qualify them for inclusion in this trial. Questions focused on their medical conditions and medications, to explore the perceived meaning and impacts of these in their daily lives. From this experience, we developed different patient phenotypes or archetypes, which was foundational to developing the intervention [27].

Step 3-tailoring the intervention: beyond these patient archetypes, we used several individual measures to tailor the intervention for individuals' situation. (1) The Necessity-Concerns framework [28] was used to personalize the intervention to individuals' circumstances and beliefs. We stratified individuals into categories of necessity-predominant beliefs (those who don't believe their medications are necessary or helpful) and concerns-predominant beliefs (those who have concerns about side-effects, dependency or interactions). (2) Given that different types of messaging might be required for those who are non-adherent to their medications, we used validated instruments for assessing adherence at study baseline (Morisky Adherence Questionnaire) [29] to assist in categorizing participants appropriately.

Step 4-procuring patient recommendations: we searched publicly accessible websites from professional societies and patientinformation groups to obtain the medical information appropriate for trial participants. These included dietary and physical activity recommendations, scientifically-sound explanations of medication effects, and evidence of medication efficacy. These materials were reviewed by clinical committee members for appropriateness for inclusion.

Step 5-brand and message development: based on insights generated from the immersive patient research, the marketing team created a patient-facing brand and visual identity for the engagement platform. They then transformed physician-vetted medical information into messages that are easily understandable and relatable by the majority of potential participants. Specific messaging was developed to address each different patient archetype, adherence-state and beliefs-state described above.

Step 6-development of the online platform: the marketing team in conjunction with Locus Health, the health technology partner, then designed, developed and built an online portal for patients to interface with the design elements and messaging material developed in step 5. Participants who indicate a preference for an electronic mode of communication receive an email on a daily basis inviting them into their portal where they receive information, or are asked to respond to as series of cards. For each card completed, participants receive points which are tracked and make them eligible to receive virtual awards. This approach is designed to improve engagement with the platform through positive reinforcement.

Step 7-development of the analog platform: from previous studies in the area, we expected that at least half of study participants would not be able or willing to access the online platform [30]. From the types of messaging developed in step 5, our marketing partners developed paper-based mailers that participants randomized to receive the self-management intervention receive weekly focusing on medication adherence and positive health behavior choices.

Step 8-development of the facilitated relay strategy: since only $50 \%$ of Albertans with chronic diseases are taking guidelinerecommended therapies $[10,31]$, we developed personalized letters that would be sent to participants to take to their primary care 
provider and to their pharmacist. Where individuals are not currently prescribed both classes of recommended medications, the purpose of the letter is to remind the care provider about the evidence behind these therapies and strategies to attempt if the drugs had previously not been tolerated. For those who are on both statins and ACEi/ARBs at baseline, the letter simply informs their provider that they are enrolled in a clinical trial with a self-management intervention designed to assist them in working with the patient for improved heart health. These letters were pilot-tested with five primary care physicians and four pharmacists and refined based on the feedback received.

Step 9-focus group testing: once a preliminary version of the intervention was designed, we convened two focus groups of individuals ( $n=6$ and $n=8$ ) who would meet inclusion criteria for the trial. We solicited their feedback about the various elements of the intervention (including the brand identity and visual design), receiving important comments and suggestions. For example, we heard that our initial design was too complex and that navigating the site was not intuitive. We also heard that the brand name of the platform was unpalatable and not easily relatable to focus group participants.

Step 10-intervention redesign: based on the feedback received in step 9, the marketing and health technology teams made further changes to the delivery platform. In response to feedback about overly complex design, the intervention was simplified and streamlined to increase usability. The platform was also renamed and rebranded as a result of focus group feedback.

Step 11-beta testing: 20 individuals were recruited to beta test the platform, including members of the study team, as well as older adults with chronic diseases. Users were given access to the electronic platform for a two-week period at which point all users were asked to complete a debriefing questionnaire, and qualitative debriefing interviews were undertaken with participating seniors. Step 12-finalization of the components and implementation strategy for intervention 2: after completion of the above steps, we finalized the self-management intervention and describe below an overview of the patient experience:

Week 1 -participants receive a letter informing them that they have been randomized to receive the personalized self-management intervention.

Week 2-participants receive a designed, personalized starter kit welcoming them to the informational platform, including:

- Information guide booklet with information on how to log in to the online platform

- Quick start guide

- Pedometer

- Wallet card to record user name and password

- Facilitated relay letters to take to primary care physician and pharmacist, tailored based on whether the patient is receiving statins and/or ACEi/ARB at baseline, and if not, whether they have tried them previously and experienced side-effects or had significant medicationrelated concerns as identified on their responses to the Beliefs about Medications Questionnaire. For patients not receiving either statins or ACEi/ARB, the initial letter focuses on statin use, with a subsequent letter about ACEi/ARB use sent at 3 months.

Daily throughout study — participants receive an email with personalized messages around medication, adherence, relevant health behaviors or their specific chronic conditions. These emails encourage them to log in to their secure online portal for further information.

NOTE: those who choose to receive their intervention through non-electronic means receive weekly mailers instead of daily emails. At 1 month-participants receive a letter reminding them to take their initial facilitated relay letters to their physician and pharmacist. Monthly_all participants receive paper-based mailers through the postal mail system. These are designed to drive engagement with the platform and contain general information about healthy living, including anecdotes, recipes, and suggestions.

At 3 months - to decrease the risk of disengagement, participants receive a free gift (a branded re-useable shopping bag) thanking them for participating and encouraging them to continue.

At 3 months - participants who were not on either statins or ACEi/ARBs at baseline receive their second set of facilitated relay letters (on the importance of ACEi/ARB use) to take to their healthcare providers.

At 6 months - participants receive a menu in the mail inviting them to select one of two free gifts, which include a new upgraded pedometer, or a tailored and branded health tracking book.

At 6 months-participants also complete their 6-month follow-up survey, including updated information on medication use and adherence-which will inform future interactions with the platform.

Daily/weekly/monthly interactions continue through the duration of the trial. 


\section{Control arms}

The control arm for intervention 1 (copayment elimination) is the usual pharmaceutical insurance plan. Those who are randomized to usual insurance coverage continue to have access to their regularly prescribed medications through their Alberta Blue Cross seniors plan with their usual $30 \%$ copayment to a maximum of $\$ 25$ per prescription.

The control for intervention 2 (the self-management intervention) has access to generic chronic disease information. Participants in this study arm are provided with a link to a government website (myhealth.alberta.ca) housing health information on a variety of conditions written by the company Healthwise. Participants can find information that interests them on a self-directed basis. They are also automatically subscribed to receive the quarterly patient-focused magazine published by Alberta Health Services.

\section{Outcomes}

The primary outcome is the composite rate of all-cause mortality, MI, stroke, coronary revascularization (coronary artery bypass grafting, angioplasty, or coronary stenting), and hospitalizations for cardiovascular-related ambulatory care-sensitive conditions defined using validated algorithms through administrative data [16] (Additional file 1: Appendix 2). As an individual may experience multiple events, a rate outcome will be used rather than a binary composite. Secondary outcomes include: (1) individual components of the primary endpoint, (2) changes to medication adherence, medication self-efficacy and needsconcerns states (see below), and (3) overall quality of life. Alongside the clinical trial, we are also planning a costeffectiveness analysis, and a qualitative descriptive study, both described briefly in separate sections below.

\section{Sample size}

We identified a cohort of 170,000 Alberta seniors from the Interdisciplinary Chronic Disease Collaboration (ICDC) Chronic Disease Repository (Additional file 1: Appendix 3) who would meet study entry criteria. We found that the annual composite primary outcome event rate was estimated as 14 per 100 participant years. We used this rate to determine sample size requirements [32], assuming a minimal clinically important relative risk reduction of $12, \alpha=5 \%, 80 \%$ power, allocation ratio of 1:1, average follow up of 3 years and presumed 1 percent per year loss to follow-up (due to outmigration from Alberta) [33]. Also, 4714 patients are required, assuming no important interaction between our two interventions, which was tested using simulations with plausible interactions of 25 and $50 \%$.

\section{Recruitment strategies}

Participants will be recruited via signage in primary care and specialist physician offices as well as in pharmacies. Several hundred pharmacies across the province have agreed to participate in recruitment for this trial by posting study posters and by handing recruitment brochures to any patients filling medications that would suggest they might be eligible for this study (anti-hypertensive or anti-diabetes medications). We will also recruit using local newspapers and community newsletters, through senior's groups as well as mass media (television, radio and social media).

\section{Sequence generation and allocation concealment}

Once baseline surveys have been returned to the study team and we have verified eligibility, randomization to the interventions will occur in a 1:1:1:1 fashion via a computer generated randomization program.

A randomized treatment allocation table was created on October 28, 2015 using sealedenvelope.com. Four treatment groups were used (intervention 1 only, intervention 2 only, interventions 1 and 2, and no intervention/control). Variable block sizes were used and the list length was 7200 . Randomization was stratified by age ( $<70$ years, $\geq 70$ years); income $(<\$ 30,000, \geq \$ 30,000)$; and sex (Male, Female).

Study data will be collected and managed using REDCap electronic data capture tools hosted at the University of Calgary, in collaboration with the Cumming School of Medicine. The randomization module was enabled in REDCap, and the allocation table produced by Sealed Envelope was imported into the web-based application. Patients will be entered into the database and randomized, in a concealed fashion, according to this table by the study coordinating centre.

\section{Blinding}

Given the pragmatic nature of copayment elimination, blinding is not possible for this intervention. For the selfmanagement intervention, even control patients will receive a basic version of potentially relevant educational materials, in essence being single-blinded for this part of the study.

However, to deal with the risk of ascertainment bias in this observational study, administrative data codes defining the primary and secondary outcomes were defined in advance (Additional file 1: Appendix 2) and outcome assessment will be blinded by having an analyst who does not have access to treatment information.

\section{Data collection}

Subjective outcomes such as quality of life, self-efficacy, and needs-concerns will be obtained from surveys administered at baseline, 6, 18, and 36 months (study completion). Objective outcomes will be assessed using the ICDC Chronic Disease Repository [32], which 
includes provincial laboratory and administrative health data (including vital statistics, pharmacy claims, physician claims, hospitalizations, emergency department and outpatient visits, and all health care costs) for all Albertans (Additional file 1: Appendix 3). This dataset has been used for many observational studies [34-37] and for assessing outcomes in a RCT of over 20,000 individuals [33].

Primary endpoint: the use of administrative databases to identify clinical conditions and outcomes has been validated in numerous high-quality studies [38-40], and the specific ICD-10 codes we have chosen to define the outcomes have been endorsed by respected national organizations [41] and based on validated algorithms applied to administrative health data [38, 40, 42-47].

Secondary endpoints:

(1) Medication adherence: measured by the "number of days dispensed"/"number of days between prescription renewals" $[48,49]$ using drug data in the ICDC Chronic Disease Repository. Patients with medication supplies to cover $\geq 80 \%$ of observed treatment days are considered adherent $[21,50]$.

(2) Quality of life: we will administer the Euroqol EQ5D-5 L [51-53] at 6, 18, and 36 months [54]. The Canadian-specific EQ5D index score will be the primary quality of life measure [55].

(3) Medication self-efficacy: we will administer the validated MASES questionnaire [56] at baseline and at study completion.

(4)Necessity-concerns: we will administer the validated BMQ-specific questionnaire [57] at baseline and at study completion.

\section{Data management}

Data entry for paper surveys will be done using iDataFax (DF/Net Research Inc I, Seattle, WA) with duplicate verification. All data will be stored in the study's central data repository on secured University of Calgary servers within the Clinical Research Unit. Given the low-risk nature of our interventions, and since our outcomes will be assessed using administrative data (with a one-year lag to receipt of data), there will be no external data safety and monitoring board. This study is considered low risk since patients' physicians remain ultimately responsible for managing patients' medical treatments and any complications that may arise as part of their treatment.

\section{Statistical analysis}

Primary outcome:

A Poisson model will test the main effects of the impact of the interventions on the rate of the primary outcome. This technique was chosen as individuals may experience multiple outcomes prior to the end of the study period, therefore we will account for both number of events and varying observation time within the Poisson model. The likelihood ratio test will test a negative binomial regression model within the Poisson model to examine for the presence of over dispersion. If present, negative binomial models will be used. The Vuong test will examine for excess zeros [58]. If present, zero-inflated models will be used.

Secondary outcomes:

Medication adherence is a binary variable and will be analyzed using log binomial regression (generalized linear models with a log link) -given the likely high prevalence of non-adherence. EQ5D index scores are continuous and will be analyzed using linear regression. Medication self-efficacy and concerns will be dichotomized and analyzed using logistic regression.

All analyses will be done using the "intention to treat" principle. Subgroup analyses will be based on underlying chronic disease and income status. Multiple imputations will be used to handle missing data.

\section{Cost-effectiveness analysis}

Alongside this randomized trial, we will conduct a costutility analysis using the EQ5D index scores collected within the trial and administrative data sources to obtain costing information.

\section{Background}

Using the cohort of 170,000 Albertans noted above, cost of health care (physician, hospitalization, ambulatory care, and drug costs) was noted to exceed $\$ 12,000$ per patientyear, with $\sim 35 \%$ of these costs related to components of the primary outcome (Table 1). We will assess whether reductions in the overall costs of care offset the cost of copayment elimination, or enhanced self-management education.

\section{Objective}

The objective is to determine the cost per quality adjusted life year gained for the two study interventions in low-income seniors with chronic diseases.

\section{Methods}

We will calculate the total costs of care for patients randomized to each of the interventions. Using administrative data, the cost of physician visits, emergency department visits, chronic disease-related hospitalizations, outpatient interventions related to chronic diseases and medications will be calculated for each participant. Alberta Health uses CIHI case mix grouper methods and ambulatory case costing methods to estimate hospital and outpatient costs, respectively. Physician claims will be based on the amount paid by Alberta Health [59, 60]. If needed, extrapolation over a patient's lifetime will be made using decision analysis $[59,60]$. 
Table 1 Healthcare costs over a three-year period for a cohort of 170,000 Albertans noted as potentially eligible for this study using administrative health data

\begin{tabular}{|c|c|c|c|c|}
\hline & $\begin{array}{l}\text { No. of patients with an event } \\
\text { within primary composite } \\
\text { endpoint, or physician claim }\end{array}$ & $\begin{array}{l}\text { Total events (including } \\
\text { recurrent events) or } \\
\text { physician claims }\end{array}$ & $\begin{array}{l}\text { Percentage of patients } \\
\text { with an event or } \\
\text { physician claim }\end{array}$ & $\begin{array}{l}\text { Average cost per patient over } \\
\text { three years (includes those } \\
\text { without any events) }\end{array}$ \\
\hline Hospitalization events & 47,201 & 110,773 & 48.7 & $\$ 18,417$ \\
\hline Physician claims & 95,018 & $7,765,483$ & 98.4 & $\$ 5377$ \\
\hline $\begin{array}{l}\text { Ambulatory care / emergency } \\
\text { department encounters }\end{array}$ & 85,105 & $1,594,117$ & 88.1 & $\$ 5200$ \\
\hline Prescription drug claims or costs & 88,247 & $10,944,192$ & 91.4 & $\$ 5915$ \\
\hline $\begin{array}{l}\text { Sum of all above costs } \\
\text { over } 3 \text { years }\end{array}$ & & & & $\$ 36,332$ \\
\hline
\end{tabular}

\section{Qualitative descriptive study \\ Overview}

Qualitative research methods will be used to explore which intervention components did or did not work, why and in what contexts, by eliciting participants' views and their reasons for non-adherence or adherence to self-management recommendations (including adherence to medications).

\section{Sampling}

A purposive sampling strategy will reflect: sex; type of chronic condition; income; and perception of a financial barrier. Five to ten participants will be selected from each of three active study arms (co-payment elimination only, self-management only, and both) across four groups of interest (those not on statins at baseline who started statins, those not on statins at baseline who remained off statins, those on statins at baseline with poor adherence but whose adherence improved, and those on statins at baseline with poor adherence and no improvement) resulting in a total of 12 groups. Sampling will continue to saturation, which we expect to occur at between 60-120 interviews.

\section{Data collection}

Telephone interviews will be conducted by a trained research assistant six months after enrolment to explore: general perceptions as to whether the intervention addressed individuals' barriers; which aspects participants found helpful or not; why the intervention was successful at changing individual health behaviors or not; how the intervention affected individual's quality of life (positively or negatively) and how the intervention could have been more impactful.

\section{Data analysis}

Qualitative descriptive/thematic analysis will be undertaken using qualitative data analysis and management software. Initial microcoding will break transcribed data into granular concepts, with subsequent coding to group concepts into themes. All analyses will be performed in duplicate by experienced qualitative data analysts.

\section{Impact of qualitative study}

In addition to gaining a better understanding of why the intervention(s) did or did not work, the results will be used to refine the self-management intervention that is provided in the remainder of the study, and will provide explanatory power for trial findings, especially if the interventions do not improve adherence and health outcomes.

\section{Discussion}

Given the frequency of financial barriers to medication use in low-income Albertans, this study will test the impact of a value-based formulary which eliminates copayment for high-value preventive medication on outcomes and costs. Given the frequency of knowledge and motivation-related barriers, we will also assess the impact of a tailored self-management education and facilitated relay strategy which, if effective, could be rolled out in a cost-efficient manner to primary care clinics that have rosters of patients with chronic diseases. By comprehensively measuring the impact of the interventions on both health outcomes and costs, as well as the impact on reducing health inequities in this vulnerable population, our study will facilitate informed formulary policy decisions.

Many interventions that seek to change patient behavior have not been successful at improving adherence or improving outcomes $[61,62]$, so our team decided to take a different approach by incorporating input from both patients and commercial marketing/design firms in the intervention development phase. We believe that this novel academic, industry, and patient co-design approach will provide a framework for future initiatives.

By comprehensively measuring the impact of the interventions on both health outcomes and costs, as well as the impact on reducing health inequities in this vulnerable population, policy-maker partners will have all the information required to make informed formulary policy decisions.

We will present our findings at national and international conferences on health policy and drug financing. We aim to publish our findings in academic general medical journals. Authorship will be determined 


\section{by the International Committee of Medical Journal Editors (ICMJE) criteria for authorship.}

\section{Additional file}

Additional file 1: Appendix 1. Medications included in copayment elimination intervention. Appendix 2. ICD10-CA definitions of the components of our primary clinical endpoint. Appendix 3. Alberta Kidney Disease Network Administrative Database. Appendix 4. Informed consent form. (DOCX $131 \mathrm{~kb}$ )

\section{Acknowledgements}

Not applicable.

\section{Funding}

The study is funded by Alberta Innovates-Health Solutions, the University of Calgary Clinical Research Fund, and the Canadian Institutes of Health Research (through an investigator Foundation grant (Manns)). The funding partners and trial sponsor had no input into the study design or data collection methods. They will not have access to trial data or any input into analysis or interpretation of results, or writing of the report, including decision to submit for publication. Alberta Innovates-Health Solutions, University of Calgary Clinical Research Fund Canadian Institutes of Health Research Foundation Grant (Manns). Composition, roles and responsibilities of study committees: Study Coordinating Centre: Terry Saunders-Smith, Sharon Gulewich, Nicole MacDonald, Alexa Desjarlais.

Steering Committee: Braden Manns, Brenda Hemmelgarn, Marcello Tonelli, David Campbell, Chad Mitchell, Ross Tsuyuki.

Endpoint Adjudication Committee: Derek Exner, Peter Faris.

Data Management Team: Peter Faris, Jianguo Zhang, Terry Saunders-Smith, Sharon Gulewich.

\section{Availability of data and materials}

The full study protocol will be made available on our website (ICDC.ca) and statistical code will be made available at the time of publication; however, the participant-level dataset will not be made publicly available due to restrictions set by our data access agreement.

\section{Authors' contributions}

$\mathrm{DC}$ is a co-investigator and primary proposal author, and will conduct study analyses, along with PF. MT, BH, RT, NI, TC, SK, KK-S, PF, DE, and VC helped design the study, and provided key revisions to the study protocol. CM was a liaison with the Alberta government, ensuring key input on the value-based formulary, and providing key revisions to the study protocol. PF designed the statistical analysis, and provided key revisions to the study protocol. RP and EV helped design intervention 2, and provided key revisions to the study protocol. BM is the principal investigator, developed the study idea with DC and the other authors, acquired study funding along with $\mathrm{BH}$, and $\mathrm{MT}$, and wrote and revised sections

of the final protocol presented herein. All authors read and approved the final manuscript.

\section{Competing interests}

The authors declare that they have no competing interests.

\section{Consent for publication}

Not applicable.

\section{Ethics approval and consent to participate}

We have received approval from our institutional review board (University of Calgary Conjoint Health Research Ethics Board (CHREB) \#: 13-1241; University of Alberta Health Research Ethics Board \#PRO00062473). Protocol modifications will be decided upon by the steering committee and will need to be approved by CHREB prior to implementation. In cases where such modifications may affect a participant's intervention or data, they will be notified in writing of the proposed changes prior to implementation.

Informed consent to participate in the ACCESS trial is received via a two-step process: initially, consent to participate in the telephone screening interview is obtained verbally. Once eligibility is confirmed, study information packages are sent (electronically or in the post) to potential participants to obtain written informed consent (Additional file 1: Appendix 4). Proxy consent is permitted for participants who do not speak English fluently and who have a close family member or friend who will agree to read consent forms to the participant in their native language. Explicit consent will be obtained for linkage to participants administrative health data records.

Confidentiality will be assured through the use of a unique dual data capture system. Identifiable information (name, date of birth, personal health number) will only be stored on the secured, encrypted server. By contrast, all surveys and data collection instruments are sent from an anonymized twinned version of this dataset. Unique study identification numbers will be used to permit linkage from the anonymized to the secure side of the database

\section{Author details}

${ }^{1}$ Departments of Medicine and Community Health Sciences, Cumming School of Medicine, University of Calgary, Calgary, Canada. ${ }^{2}$ Departments of Medicine and Community Health Sciences, Libin Institute and Institute for Public Health, Cumming School of Medicine, University of Calgary, Calgary, Canada. ${ }^{3}$ Alberta Health, Edmonton, Canada. ${ }^{4}$ Department of Pharmaceutical Sciences, University of Alberta, Edmonton, Canada. ${ }^{5}$ Department of Family Medicine, University of Toronto, Toronto, Canada. ${ }^{6}$ Department of Psychology, University of Calgary, Calgary, Canada. ${ }^{7}$ Emergence Creative, New york, USA. ${ }^{8}$ Department of Medicine, University of Alberta, Edmonton, Canada. ${ }^{9}$ Faculty of Nursing, University of Calgary, Calgary, Canada. ${ }^{10}$ Alberta Health Services, Edmonton, Canada. ${ }^{11}$ Department of Medicine, University of British Columbia, Vancouver, Canada. ${ }^{12}$ Foothills Medical Centre, 1403 29th St. NW, Calgary, Alberta, CanadaT2N 2T9.

Received: 10 August 2016 Accepted: 8 September 2016

Published online: 26 September 2016

\section{References}

1. World Health Organization. Cardiovascular diseases. http://www.who.int/ cardiovascular_diseases/en/. Accessed 5 Jul 2016.

2. Statistics Canada. Mortality, summary list of causes. Ottawa: Statistics Canada; 2011

3. Leibowitz A, Manning WG, Newhouse JP. The demand for prescription drugs as a function of cost-sharing. Soc Sci Med. 1985;21:1063-9.

4. Keeler EB, Brook RH, Goldberg GA, Kamberg CJ, Newhouse JP. How free care reduced hypertension in the health insurance experiment. JAMA. 1985:254:1926-31.

5. Tamblyn R, Laprise R, Hanley JA, Abrahamowicz M, Scott S, Mayo N, et al. Adverse events associated with prescription drug cost-sharing among poor and elderly persons. JAMA. 2001;285:421-9.

6. Psaty BM, Lumley T, Furberg CD, Schellenbaum G, Pahor M, Alderman MH et al. Health outcomes associated with various antihypertensive therapies used as first-line agents: a network meta-analysis. JAMA. 2003;289:2534-44.

7. Gaede $P$, Vedel P, Parving HH, Pedersen O. Intensified multifactorial intervention in patients with type 2 diabetes mellitus and microalbuminuria: the Steno type 2 randomised study. Lancet. 1999:353:617-22.

8. Nelson KM, McFarland L, Reiber G. Factors influencing disease selfmanagement among veterans with diabetes and poor glycemic control. J Gen Intern Med. 2007:22:442-7.

9. Barnieh L, Clement F, Harris A, Blom M, Donaldson C, Klarenbach S, et al. A systematic review of cost-sharing strategies used within publicly-funded drug plans in member countries of the organisation for economic cooperation and development. PLoS One. 2014;9:e90434.

10. Campbell D, King-Shier K, Hemmelgarn B, Sanmartin C, Ronksley P, Weaver $R$, et al. Assessing the association of financial barriers on care and outcomes for patients with chronic disease. Health Rep. 2014;25.

11. Alberta Blue Cross. Plans for individuals and families. https://www.ab. bluecross.ca/individuals-families/index.html Accessed. 5 Jul 2016.

12. Daw JR, Morgan SG. Stitching the gaps in the Canadian public drug coverage patchwork? A review of provincial pharmacare policy changes from 2000 to 2010. Health Policy. 2012;104:19-26.

13. Interdisciplinary chronic disease collaboration. Barriers to Care for People with Chronic Health Conditions Survey. http://www.icdc.ca/research/ selected-projects\#5-barriers-to-care-for-people-with-chronic-healthconditions-bcpchc-survey Accessed. 5 Jul 2016.

14. Bodenheimer $\mathrm{T}$, Lorig $\mathrm{K}$, Holman $\mathrm{H}$, Grumbach $\mathrm{K}$. Patient self-management of chronic disease in primary care. JAMA. 2002;288:2469-75. 
15. Ansari Z, Laditka JN, Laditka SB. Access to health care and hospitalization for ambulatory care sensitive conditions. Med Care Res Rev. 2006;63:719-41.

16. Canadian Institute for Health Information (CIHI). Technical note: ambulatory care sensitive conditions (ACSC). www.cihi.ca/ClHI-ext-portal/pdf/internet/ DEFINITIONS 052010_EN Accessed. 5 Jul 2016.

17. Friedman B, Basu J. The rate and cost of hospital readmissions for preventable conditions. Med Care Res Rev. 2004;61:225-40.

18. Booth G, Hux J. Relationship between avoidable hospitalizations for diabetes mellitus and income level. Arch Intern Med. 2003;163:101-6.

19. Campbell D, Ronksley P, Barnabe C, Tonelli M, Hemmelgarn B, Manns B. The association of enrolment in primary care networks on diabetes care and outcomes in low income and first nations Albertans. Open Med. 2012;6:E155-65.

20. Tang KL, Bamieh L, Mann B, Clement F, Campbell DJT, Hemmelgarn BR, et al. A systematic review of value-based insurance design in chronic diseases. Am J Manag Care. 2014;20:E229-40.

21. Choudhry NK, Avorn J, Glynn RJ, Antman EM, Schneeweiss S, Toscano M, et al. Full coverage for preventive medications after myocardial infarction. N Engl J Med. 2011;365:2088-97.

22. Tricco AC, Ivers NM, Grimshaw JM, Moher D, Turner L, Galipeau J, et al. Effectiveness of quality improvement strategies on the management of diabetes: a systematic review and meta-analysis. Lancet. 2012:379:2252-61.

23. Serrano V, Rodriguez-Gutierrez R, Hargraves I, Gionfriddo MR, Tamhane S, Montori VM. Shared decision-making in the care of individuals with diabetes. Diabet Med. 2016;33:742-51.

24. Statistics Canada. Table 051-0001 Population by year, by province and territory. Ottawa: Statistics Canada; 2015.

25. Cannon CP, Steinberg BA, Murphy SA, Mega JL, Braunwald E. Meta-analysis of cardiovascular outcomes trials comparing intensive versus moderate statin therapy. J Am Coll Cardiol. 2006;48:438-45.

26. van Vark LC, Bertrand M, Akkerhuis KM, Brugts JJ, Fox K, Mourad JJ, et al. Angiotensin-converting enzyme inhibitors reduce mortality in hypertension: a meta-analysis of randomized clinical trials of renin-angiotensin-aldosterone system inhibitors involving 158,998 patients. Eur Heart J. 2012;33:2088-97.

27. O'Connor PJ. Patient archetypes, physician archetypes, and tailored diabetes care. J Am Board Fam Pract. 2002;15:334-7.

28. Horne R, Chapman SC, Parham R, Freemantle N, Forbes A, Cooper V. Understanding patients' adherence-related beliefs about medicines prescribed for long-term conditions: a meta-analytic review of the Necessity-Concerns Framework. PLoS One. 2013;8:e80633.

29. Pedersini R, Vietri J. Comparison of the 4-item and 8-item morisky medication adherence scale in patients with type 2 diabetes. Value Health. 2014;17:A196.

30. Afshar AE, Weaver RG, Lin M, Allan M, Ronksley PE, Sanmartin C, et al. Capacity and willingness of patients with chronic noncommunicable diseases to use information technology to help manage their condition: a cross-sectional study. CMAJ Open. 2014;2:E51-9.

31. Manns BJ, Tonelli M, Zhang J, Campbell DJ, Sargious P, Ayyalasomayajula B, et al. Enrolment in primary care networks: impact on outcomes and processes of care for patients with diabetes. CMAJ. 2012;184:E144-52.

32. Hemmelgarn BR, Clement F, Manns BJ, Klarenbach S, James MT, Ravani P, et al. Overview of the Alberta kidney disease network. BMC Nephrol. 2009;10:30

33. Manns B, Tonelli M, Culleton B, Faris P, McLaughlin K, Chin R, et al. A cluster randomized trial of an enhanced eGFR prompt in chronic kidney disease. Clin J Am Soc Nephro. 2012;7:565-72

34. Hemmelgarn B, Manns B, Lloyd A, James MT, Klarenbach S, Quinn R, et al. Relation between kidney function, proteinuria, and adverse outcomes. JAMA. 2010;303:423-9.

35. Hemmelgarn BR, James MT, Manns BJ, O'Hare AM, Muntner P, Ravani P, et al. Rates of treated and untreated kidney failure in older vs younger adults. JAMA. 2012;307:2507-15.

36. Hemmelgarn BR, Zhang JG, Manns BJ, James MT, Quinn RR, Ravani P, et al. Nephrology visits and health care resource use before and after reporting estimated glomerular filtration rate. JAMA. 2010;303:1151-8.

37. Tonelli M, Muntner P, Lloyd A, Manns BJ, Klarenbach S, Pannu N, et al. Risk of coronary events in people with chronic kidney disease compared with those with diabetes: a population-level cohort study. Lancet. 2012;380:807-14.

38. Gao S, Manns BJ, Culleton BF, Tonelli M, Quan H, Crowshoe L, et al. Access to health care among status aboriginal people with chronic kidney disease. CMAJ. 2008:179:1007-12
39. Hux JE, Ivis F, Flintoft V, Bica A. Diabetes in Ontario: determination of prevalence and incidence using a validated administrative data algorithm. Diabetes Care. 2002;25:512-6.

40. Quan H, Khan N, Hemmelgarn BR, Tu K, Chen G, Campbell N, et al. Validation of a case definition to define hypertension using administrative data. Hypertension. 2009:54:1423-8.

41. Canadian Institute for Health Information (ClHI). Technical Note: ambulatory care sensitive conditions. http://www.cihi.ca/CIHI-ext-portal/internet/en/ document/health+system+performance/indicators/health/tech_acsc_2011 Accessed. 5 July 2016.

42. Lee DS, Donovan L, Austin PC, Gong Y, Liu PP, Rouleau JL, et al. Comparison of coding of heart failure and comorbidities in administrative and clinical data for use in outcomes research. Med Care. 2005:43:182-8.

43. Austin PC, Daly PA, Tu JV. A multicenter study of the coding accuracy of hospital discharge administrative data for patients admitted to cardiac care units in Ontario. Am Heart J. 2002;144:290-6.

44. Kokotailo RA, Hill MD. Coding of stroke and stroke risk factors using international classification of diseases, revisions 9 and 10. Stroke. 2005;36:1776-81.

45. Quan H, Parsons GA, Ghali WA. Validity of information on comorbidity derived rom ICD-9-CCM administrative data. Med Care. 2002;40:675-85.

46. Robitaille C, Bancej C, Dai S, Tu K, Rasali D, Blais C, et al. Surveillance of ischemic heart disease should include physician billing claims: populationbased evidence from administrative health data across seven Canadian provinces. BMC Cardiovasc Disord. 2013;13:88.

47. Quan H, Sundararajan V, Halfon P, Fong A, Burnand B, Luthi JC, et al. Coding algorithms for defining comorbidities in ICD-9-CM and ICD-10 administrative data. Med Care. 2005:43:1130-9.

48. Choudhry NK, Shrank WH, Levin RL, Lee JL, Jan SA, Brookhart MA, et al. Measuring concurrent adherence to multiple related medications. Am J Manag Care. 2009;15:457-64.

49. Pharmacy Quality Alliance. Adherence. http://pqaalliance.org/resources/ adherence.asp Accessed. 5 July 2016.

50. Karve S, Cleves MA, Helm M, Hudson TJ, West DS, Martin BC. Good and poor adherence: optimal cut-point for adherence measures using administrative claims data. Curr Med Res Opin. 2009;25:2303-10.

51. Janssen MF, Lubetkin El, Sekhobo JP, Pickard AS. The use of the EQ-5D preference-based health status measure in adults with Type 2 diabetes mellitus. Diabet Med. 2011;28:395-413.

52. Sach TH, Barton GR, Doherty M, Muir KR, Jenkinson C, Avery AJ. The relationship between body mass index and health-related quality of life: comparing the EQ-5D, EuroQol VAS and SF-6D. Int J Obes (Lond). 2007;31:189-96.

53. Culleton BF, Walsh M, Klarenbach S, Mortis G, Scott-Douglas N, Quinn R, et al. Effect of frequent nocturnal hemodialysis vs conventional hemodialysis on left ventricular mass and quality of life: a randomized controlled trial. JAMA. 2007;298:1291-9.

54. Euroqoul. EQ-5D-5 L. http://www.euroqol.org/eq-5d-products/eq-5d-5l.html Accessed. 5 July 2016

55. Bansback N, Tsuchiya A, Brazier J, Anis A. Canadian valuation of EQ-5D health states: preliminary value set and considerations for future valuation studies. PLoS One. 2012;7:e311115.

56. Fernandez S, Chaplin W, Schoenthaler AM, Ogedegbe G. Revision and validation of the medication adherence self-efficacy scale (MASES) in hypertensive African Americans. J Behav Med. 2008;31:453-62.

57. Horne R, Weinman J, Hankins M. The beliefs about medicines questionnaire: the development and evaluation of a new method for assessing the cognitive representation of medication. Psychol Health. 1999;14:1-24.

58. Vuong $\mathrm{QH}$. Likelihood ratio tests for model selection and non-nested hypotheses. Econometrica. 1989;57:307-33.

59. McBrien KA, Manns BJ, Chui B, Klarenbach SW, Rabi D, Ravani P, et al. Health care costs in people with diabetes and their association with glycemic control and kidney function. Diabetes Care. 2013:36:1172-80.

60. Klarenbach S, Tonelli M, Pauly R, Walsh M, Culleton B, So H, et al. Economic evaluation of frequent home nocturnal hemodialysis based on a randomized controlled trial. J Am Soc Nephrol. 2013.

61. Rash JA, Cambell DJT, Tonelli M, Campbell T. A systematic review of interventions to improve adherence to statin medication: what do we know about what works? Prev Med. In press

62. Pladevall M, Divine G, Wells KE, Resnicow K, Williams LK. A randomized controlled trial to provide adherence information and motivational interviewing to improve diabetes and lipid control. Diabetes Educ. 2015;41:136-46 